\title{
IL-33 and ST2 comprise a critical biomechanically induced and cardioprotective signaling system
}

\author{
Shoji Sanada, ${ }^{1}$ Daihiko Hakuno, ${ }^{1}$ Luke J. Higgins, ${ }^{1}$ Eric R. Schreiter, ${ }^{1}$ \\ Andrew N.J. McKenzie, ${ }^{2}$ and Richard T. Lee ${ }^{1}$ \\ ${ }^{1}$ Cardiovascular Division, Department of Medicine, Brigham and Women's Hospital, Harvard Medical School, Boston, Massachusetts, USA \\ ${ }_{2}^{2}$ Medical Research Council Laboratory of Molecular Biology, Cambridge, United Kingdom.
}

\begin{abstract}
ST2 is an IL-1 receptor family member with transmembrane (ST2L) and soluble (sST2) isoforms. sST2 is a mechanically induced cardiomyocyte protein, and serum sST2 levels predict outcome in patients with acute myocardial infarction or chronic heart failure. Recently, IL-33 was identified as a functional ligand of ST2L, allowing exploration of the role of ST2 in myocardium. We found that IL-33 was a biomechanically induced protein predominantly synthesized by cardiac fibroblasts. IL-33 markedly antagonized angiotensin II- and phenylephrine-induced cardiomyocyte hypertrophy. Although IL-33 activated NF- $\kappa B$, it inhibited angiotensin II- and phenylephrine-induced phosphorylation of inhibitor of NF- $\kappa B \alpha(\mathrm{I} \kappa B \alpha)$ and NF- $\kappa B$ nuclear binding activity. sST2 blocked antihypertrophic effects of IL-33, indicating that sST2 functions in myocardium as a soluble decoy receptor. Following pressure overload by transverse aortic constriction (TAC), $\mathrm{ST}^{-/-}$mice had more left ventricular hypertrophy, more chamber dilation, reduced fractional shortening, more fibrosis, and impaired survival compared with WT littermates. Furthermore, recombinant IL-33 treatment reduced hypertrophy and fibrosis and improved survival after TAC in WT mice, but not in ST2 $2^{-/-}$littermates. Thus, IL-33/ST2 signaling is a mechanically activated, cardioprotective fibroblast-cardiomyocyte paracrine system, which we believe to be novel. IL-33 may have therapeutic potential for beneficially regulating the myocardial response to overload.
\end{abstract}

\section{Introduction}

Cardiomyocyte hypertrophy and cardiac fibrosis are common pathophysiological processes in many heart diseases, including myocardial infarction, hypertension, and valvular diseases (1-4). Traditionally, these pathophysiological changes have been considered adaptive responses to mechanical overload $(1,3,5)$ in an attempt to restore wall stresses to normal levels $(1,5)$. However, the myocardial response to mechanical overload $(2,3)$ can eventually lead to compromised contractile performance $(2,3)$ as well as lethal cardiac arrhythmias (4). Thus, understanding mechanically induced pathways in the myocardium could lead to new approaches to preventing heart failure (2-5).

It has been known for several years that ST2, an IL-1 receptor (IL-1R) family member, is basally expressed by cardiomyocytes (6). ST2 has 2 primary isoforms regulated by different promoters (7). The transmembrane ST2 isoform (ST2L) is a membrane-bound isoform with 3 extracellular IgG domains, a single transmembrane domain, and an intracellular SIR domain homologous to TLRs and other IL-1Rs. The soluble ST2 isoform (sST2) lacks the transmembrane and intracellular domains (7). Both ST2L and sST2 are biomechanically induced in cardiomyocytes (6). Furthermore, elevations of serum sST2 predict worse prognosis in patients with chronic heart failure (8) and those

Nonstandard abbreviations used: ANP, atrial natriuretic peptide; BNP, B-type

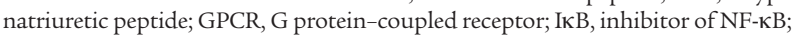
IL-1R, IL-1 receptor; MyD88, myeloid differentiation primary-response protein-88; sST2, soluble ST2 isoform; ST2L, transmembrane ST2 isoform; TAC, transverse aortic constriction.

Conflict of interest: The authors have declared that no conflict of interest exists. Citation for this article: J. Clin. Invest. 117:1538-1549 (2007). doi:10.1172/JCI30634 with acute myocardial infarction (9). The mechanisms by which ST2 signaling may affect myocardial pathophysiology have not yet been identified, in part because over a decade of searching had not yet yielded a functional ligand for ST2 $(10,11)$. However, using a structural bioinformatics approach, Schmitz et al. recently identified a novel IL-1-related sequence from a dog brain cDNA library (12). This protein, now called IL-33, proved to be a functional ligand of ST2L capable of activating NF- $\mathrm{B}$, the common signaling pathway of IL-1R/TLRs (12).

This discovery of IL-33 as a ligand for ST2 has allowed us to explore the role of IL-33/ST2 signaling in the myocardium. We hypothesized that IL-33/ST2 signaling plays a key role in regulating the myocardial response to biomechanical overload in cardiac fibroblasts and cardiomyocytes, because both cell types are mechanically activated $(3,4)$ and highly interactive $(13,14)$. Our data showed that IL-33/ST2 signaling was a biomechanically activated system that controlled cardiomyocyte hypertrophy and cardiac fibrosis. Abolishing ST2 signaling in mice led to marked cardiac hypertrophy and fibrosis following pressure overload; in addition, treatment of WT mice with purified recombinant IL-33 protected the myocardium. These data reveal IL-33/ST2 signaling as a crucial cardioprotective mechanism and provide insight into the paracrine signaling between cardiomyocytes and cardiac fibroblasts during mechanical overload.

\section{Results}

IL-33 is mechanically induced in cardiac fibroblasts and antagonizes bypertrophic stimuli. Analysis of rat neonatal cardiomyocytes and cardiac fibroblasts revealed that gene expression of IL-33 as well as sST2 was more than 5 -fold greater in cardiac fibroblasts than in cardio- 

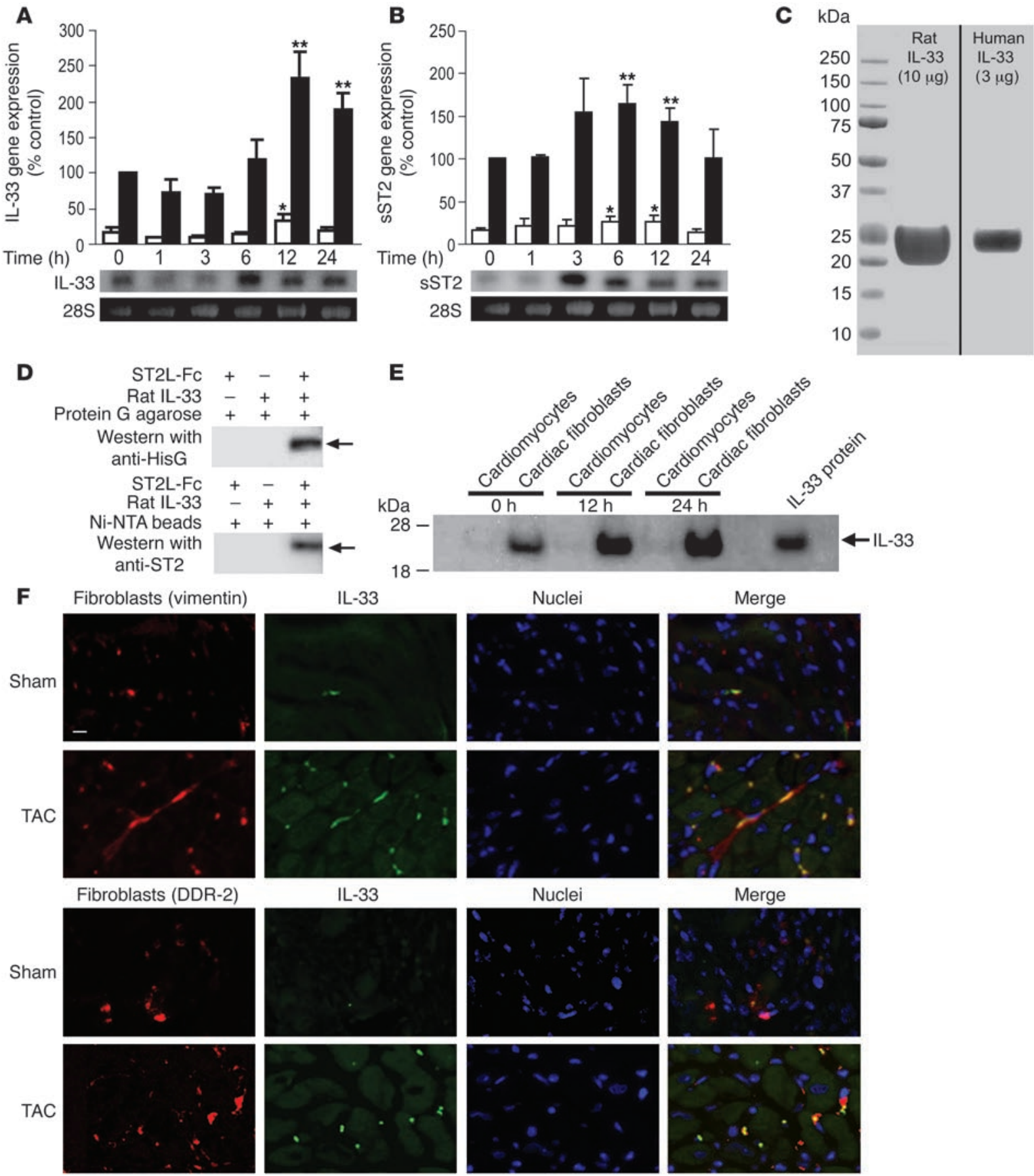

Figure 1

IL-33 is induced by mechanical strain in cardiac fibroblasts. (A and B) Quantitative analyses of gene expression of IL-33 by quantitative PCR (A) and SST2 by Northern analysis (B) in rat neonatal cardiomyocytes (white bars) and fibroblasts (black bars) are shown above with representative images from Northern analyses of cardiac fibroblast RNA. Cells were subjected to cyclic strain $(8 \%, 1 \mathrm{~Hz})$ for the indicated periods. Values are relative to $\beta$-tubulin expression and are expressed as percentage of control in cardiac fibroblasts. Data are from at least 3 sets of independent experiments. ${ }^{*} P<0.05,{ }^{* \star} P<0.01$ versus baseline. (C) Coomassie stain showed that the recombinant mature rat and human IL-33 with N-terminal His tag (10 and $3 \mu$ g protein, respectively, was loaded) were of high purity. (D) Pull-down assay of recombinant rat IL-33 with mouse ST2L-Fc protein. The recombinant protein exhibited specific binding to mouse ST2. (E) Western analysis of cardiomyocytes and cardiac fibroblasts subjected to cyclic strain (each $10 \mu \mathrm{g}$ protein sample from whole cell lysate) for the indicated periods. For reference, $0.1 \mathrm{ng}$ of recombinant IL-33 was applied in the right lane. (F) Representative immunofluorescence microscopy images of left ventricular samples 1 week after sham operation or TAC. Anti-vimentin (top panels) or anti-discoidin domain receptor-2 (DDR-2; bottom panels) antibody was used to detect fibroblasts (red) for dual staining with IL-33 (green). Pressure overload by TAC induced IL-33 expression, particularly in noncardiomyocyte interstitial cells. Scale bar: $10 \mu \mathrm{m}$. 


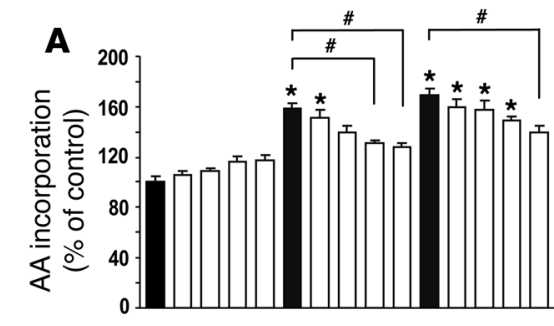

Ang II $(0.1 \mu \mathrm{M})-----+++++----$ Phe $(50 \mu \mathrm{M})$ - --------+++++ $\mathrm{IL}-33(\mathrm{ng} / \mathrm{ml})-0.1110100-0.1110100-0.1110100$
B

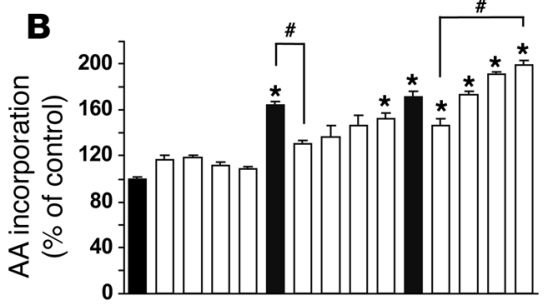

Ang II $(0.1 \mu \mathrm{M})----+++++----$

Phe $(50 \mu \mathrm{M})$ - - - - - - - - ++++++

IL-33 (ng/ml) - $10101010-10101010-10101010$ $\mathrm{sST} 2(\mu \mathrm{g} / \mathrm{ml})--0.1110--0.1110--0.1110$

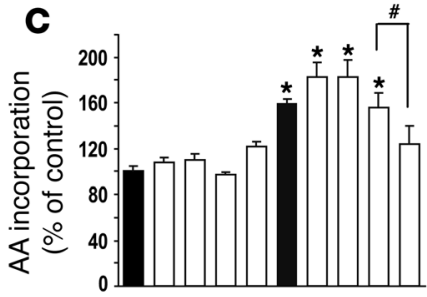

Ang II $(0.1 \mu \mathrm{M})----+++++$ $\mathrm{IL}-33(\mathrm{ng} / \mathrm{ml})--10-10--10-10$ Anti-ST2L $(\mu \mathrm{g} / \mathrm{ml})$ - $100100--100100--$ $\operatorname{lgG} 2 \mathrm{a}(\mu \mathrm{g} / \mathrm{ml})--100100--100100$

E

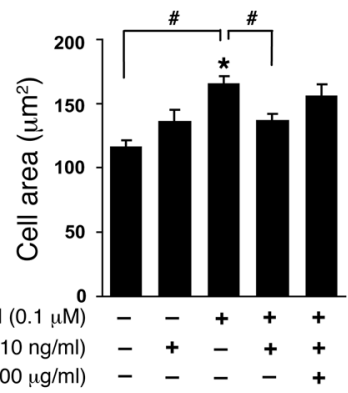

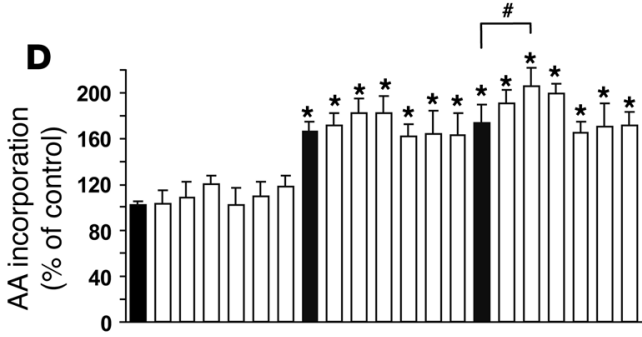

Ang II $(0.1 \mu \mathrm{M})-------+++++++-------$

Phe $(50 \mu \mathrm{M})$ - - - - - - - - - - + + +++++

$\mathrm{sST} 2(\mu \mathrm{g} / \mathrm{ml})-0.1110----0.1110----0.1110---$ IL-1Rrp2 ( $\mathrm{gg} / \mathrm{ml})----0.1110---0.1110---0.1110$

F

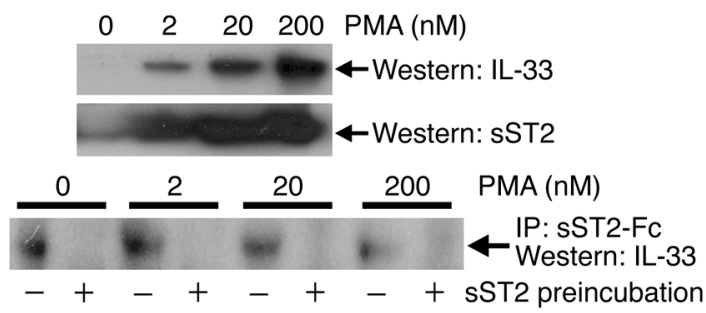

We then expressed and purified recombinant rat and human IL-33 using affinity chromatography. Both recombinant IL-33 proteins were of high purity, as both polyacrylamide electrophoresis and Coomassie staining revealed a single band (Figure 1C), and the purified IL-33 bound to the mouse ST2 protein, as demonstrated by pulldown of IL-33 using a recombinant ST2L-Fc chimera protein (Figure 1D). Endotoxin levels in the purified IL-33 were undetectable by Limulus amebocyte lysate

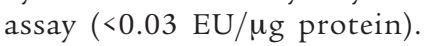
We raised a rabbit polyclonal antiserum against rat IL-33 and purified the antibodies from the antiserum by affinity chromatography. Western analysis showed that basal expression of IL-33 protein by cardiac fibroblasts was much greater than that in cardiomyocytes, and cyclic biomechanical strain $(8 \%, 1 \mathrm{~Hz})$ induced IL-33 protein in both cell types (Figure 1E). In vivo, immunofluorescent staining of mouse myocardium showed that pressure overload induced by transverse aortic constriction (TAC) increased IL-33 protein expression in cardiac interstitial cells more than in cardiomyocytes (Figure 1F). Immunofluorescence staining with antibodies to vimentin as well as discoidin domain receptor-2 (DDR-2) - which is expressed by cardiac fibroblasts but not by endothelial cells, smooth muscle cells, or cardiomyocytes $(4,15)$ - revealed nearly identical results (Figure 1F).

To determine the effects that cardiac fibroblast-secreted IL-33 might have on adjacent cardiomyocytes, cultured rat neonatal cardiomyocytes were treated with recombinant IL-33.

myocytes (Figure 1, A and B). Cyclic biomechanical strain $(8 \%, 1 \mathrm{~Hz})$ induced the expression of both IL-33 and SST2 in both cell types. In an evaluation of other stimuli, quantitative PCR showed that phorbol 12-myristate 13-acetate (PMA; $0.2 \mu \mathrm{M}$ ) and angiotensin II $(0.1 \mu \mathrm{M})$ also induced the expression of IL-33 in both cell types; in contrast, neither TNF- $\alpha(10 \mathrm{ng} / \mathrm{ml})$ nor IL- $1 \beta(10 \mathrm{ng} / \mathrm{ml})$ induced IL-33 gene expression (data not shown).
In repeated experiments, IL-33 demonstrated a modest, dosedependent prohypertrophic trend that was not statistically significant (Figure 2A). However, IL-33 potently blocked cardiomyocyte hypertrophy induced by either angiotensin II or phenylephrine in a dose-dependent manner (Figure 2A). The sST2 protein reversed the antihypertrophic effect of IL-33 in a dose-dependent manner (Figure 2B), indicating that sST2 probably functions as a soluble 


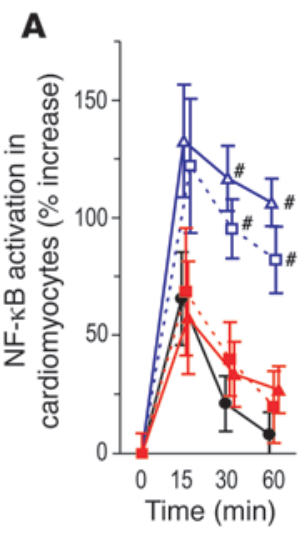

E

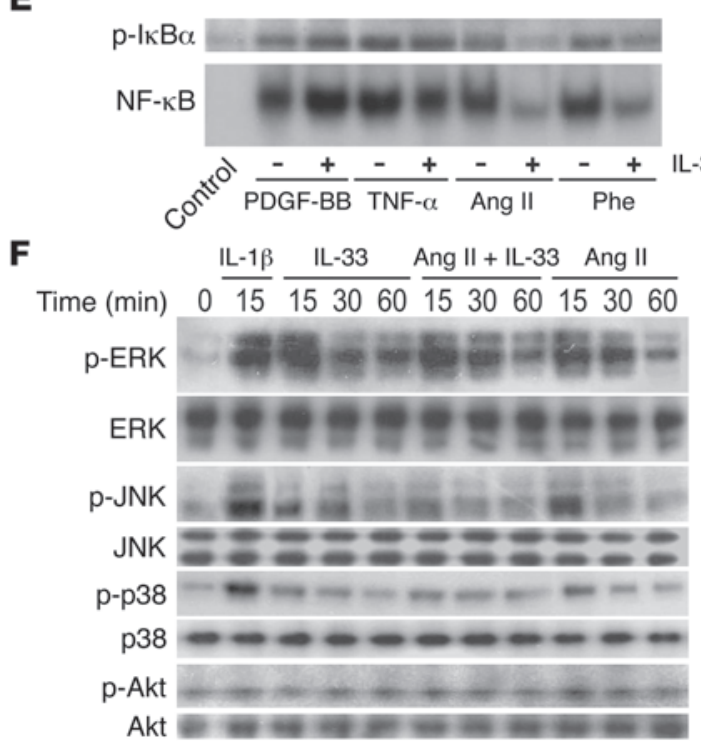

B

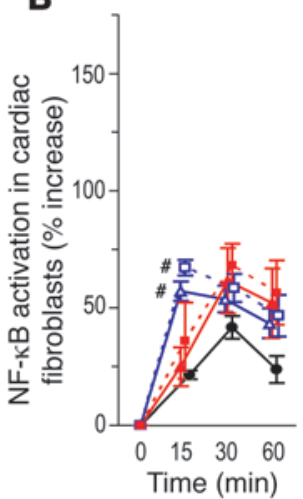

C

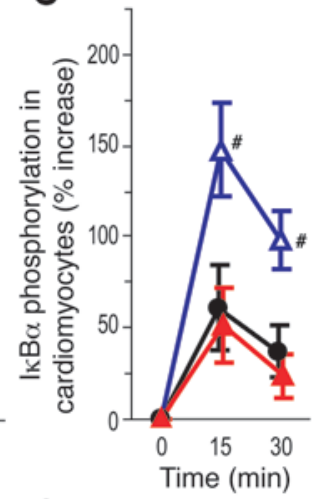

G

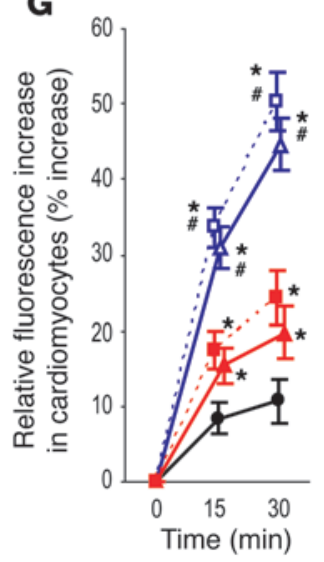

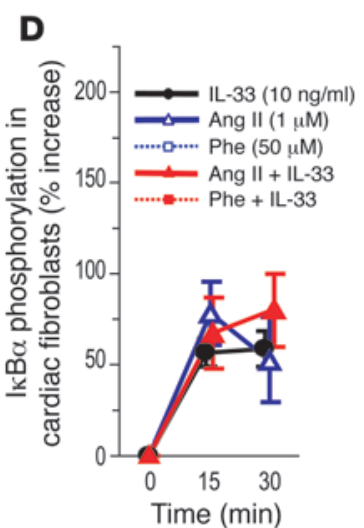

H

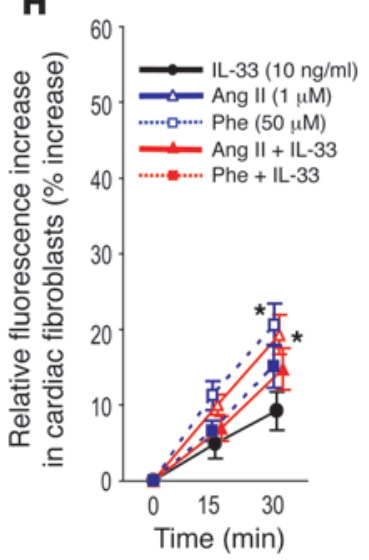

Figure 3

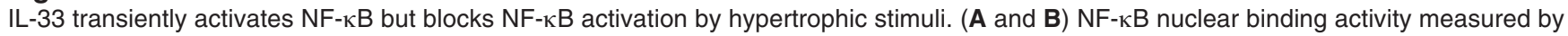
EMSA in cardiomyocytes (A) and cardiac fibroblasts (B). (C and $\mathbf{D}) \mathrm{I}_{\kappa} \mathrm{B} \alpha$ phosphorylation evaluated by Western analysis in cardiomyocytes (C) and cardiac fibroblasts (D). Values are relative to control density and are expressed as percent increase compared with control. Both angiotensin II and phenylephrine significantly activated NF-KB. IL-33 also activated NF-KB, but IL-33 markedly attenuated angiotensin II- and phenylephrineinduced NF- $\mathrm{KB}$ activation in cardiomyocytes, unlike in cardiac fibroblasts. IKB $\alpha$ phosphorylation was similarly affected by IL-33 treatment. (E)

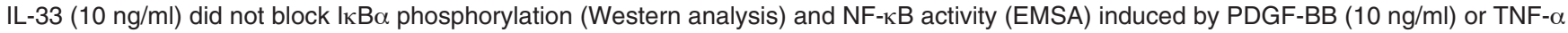
$(10 \mathrm{ng} / \mathrm{ml})$, unlike angiotensin II and phenylephrine. (F) Western analysis for MAPKs and Akt in cardiomyocytes. IL-33 (10 ng/ml) activated all MAPKs, generally to a lesser extent than did IL-1 $\beta(10 \mathrm{ng} / \mathrm{ml})$. IL-33 attenuated angiotensin II-induced phosphorylation of p38 MAPK and JNK, but not ERK or Akt. Data are from 4-5 sets of experiments. (G and H) GPCR agonist-induced ROS generation, as measured by 2,7-dichlorodihydrofluorecein diacetate, in cardiomyocytes $(\mathbf{G})$ and cardiac fibroblasts $(\mathbf{H})$. Both angiotensin II and phenylephrine significantly induced ROS generation, which was inhibited by IL-33, in cardiomyocytes. These data suggest that IL-33 can inhibit ROS-dependent hypertrophic signals. ${ }^{*} P<0.05$ versus baseline; ${ }^{\#} P<0.05$ versus the same treatment group with IL-33.

decoy receptor by binding IL-33 and preventing ST2L signaling. Blocking the ST2L receptor with an anti-ST2L monoclonal antibody eliminated the antihypertrophic effect of IL-33, unlike control IgG (Figure 2C), demonstrating that the effects of IL-33 are specifically through the ST2L receptor. In addition, the ability of SST2 to further augment the hypertrophic response under stimulation with either angiotensin II or phenylephrine was not observed with the control IL-1R-related protein 2 fusion protein (Figure 2D). The antihypertrophic effect of IL-33 was further confirmed by in vitro cell size measurement of cardiomyocytes (Figure 2E) with or without angiotensin II $(0.1 \mu \mathrm{M})$ or IL-33 $(10 \mathrm{ng} / \mathrm{ml})$ stimulation as well as application of an anti-ST2L antibody $(100 \mu \mathrm{g} / \mathrm{ml})$ that was previously reported to be a functional inhibitor of IL-33/ST2 signaling (12). The results (Figure 2E) were similar to those of the leucine uptake analysis. These data strongly suggest that IL-33 is a mechanically induced ligand for ST2L and that sST2 blocks IL-33/ST2L signaling.

High levels of sST2 can predict worse prognosis in cardiovascular patients, which suggests that SST2 levels are sufficient to sequester endogenous IL-33 levels. Despite the current lack of information on endogenous IL-33 levels in humans, we tested the hypothesis that the stimuli that increased SST2 could sequester unbound IL-33. When rat neonatal cardiac fibroblasts were stimulated with increasing doses of PMA, both sST2 and total IL-33 
A
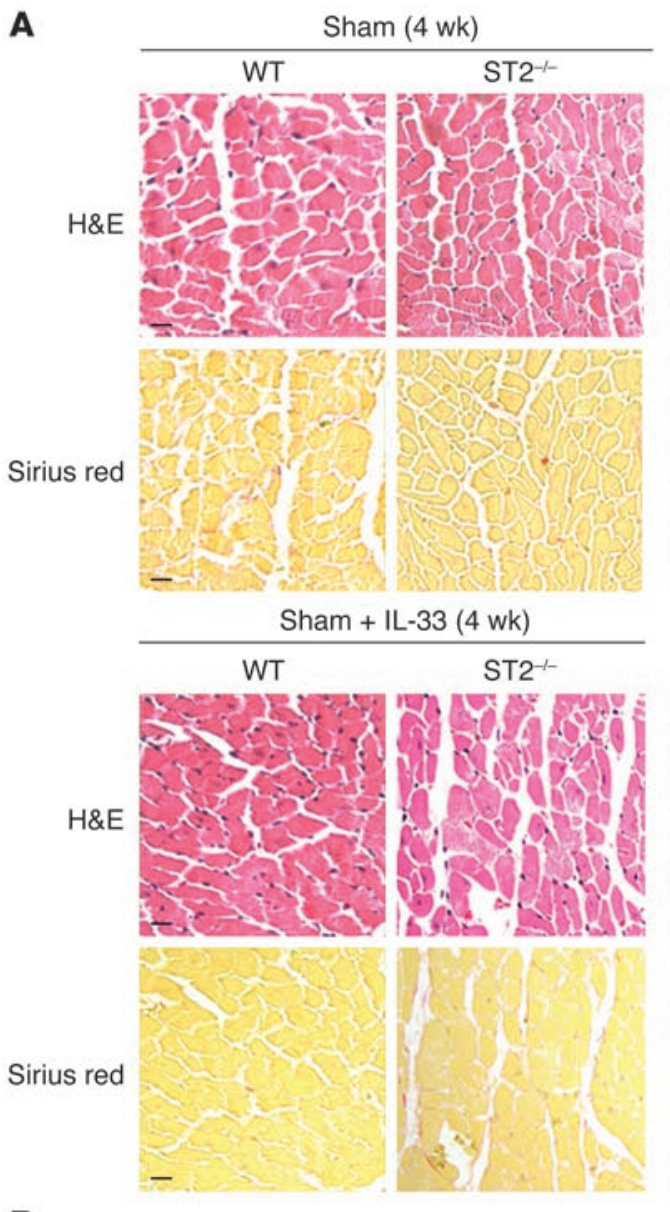

B

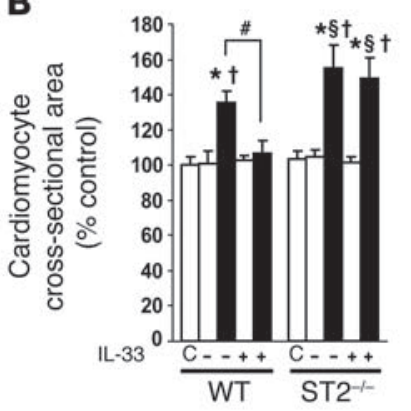

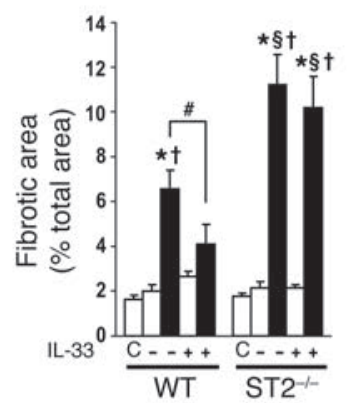
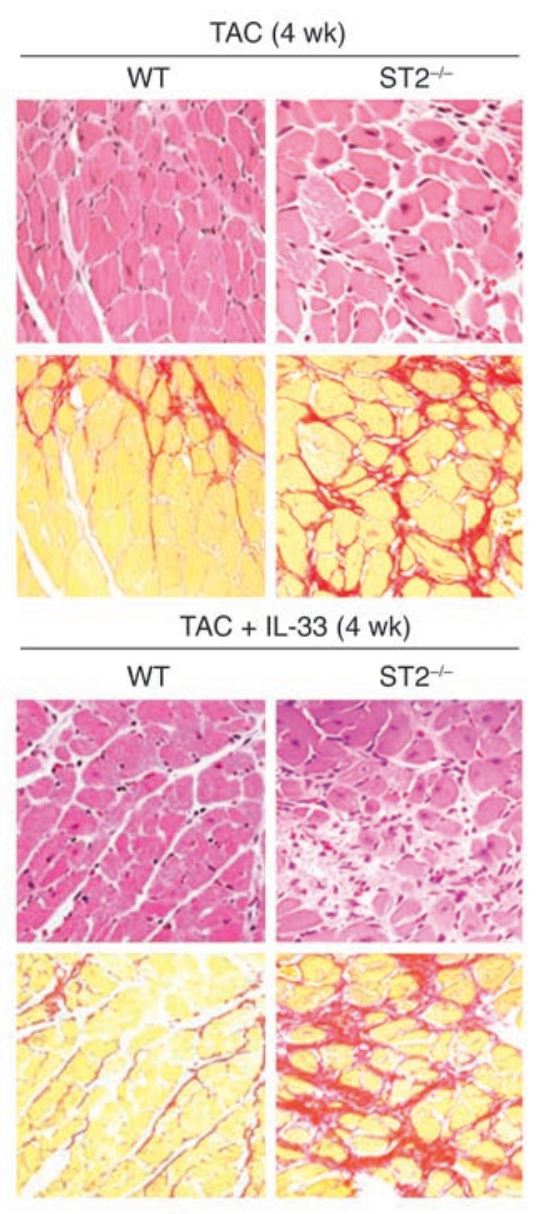

C

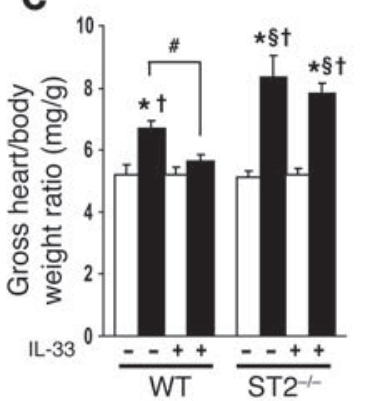

Figure 4

IL-33/ST2 signaling is cardioprotective in vivo. (A) Representative H\&E and Sirius red stains and (B) quantitative analyses of samples from each group. Computer-based image analysis was used for measurements. ST2 ${ }^{-/-}$mice developed more cardiomyocyte hypertrophy and cardiac fibrosis after TAC than did WT mice. Furthermore, treatment with IL-33 $(2 \mu \mathrm{g} / \mathrm{d}$ i.p. $)$ significantly improved these changes in WT mice, but not in ST2 ${ }^{-/}$mice. C, nonoperated control. Scale bar: $10 \mu \mathrm{m}$. (C) Gross measurement of heart weight normalized to body weight was consistent with the histomorphometric analyses. ${ }^{\star} P<0.05$ versus nonoperated control $(\mathbf{B})$ or sham-operated WT $(\mathbf{C})$; $\$$ \& $<0.05$ versus the same treatment in WT; ${ }^{\dagger} P<0.05$ versus sham in the same group; ${ }^{P} P<0.05$.

were induced (Figure 2F). However, unbound IL-33 diminished, indicating that SST2 induction could potentially limit IL-33 signaling (Figure $2 \mathrm{~F}$ ). These data suggest that the local ratio of IL-33 and SST2 could regulate IL-33 signaling.

IL-33 blocks NF-KB activation by angiotensin II and phenylephrine. Because NF- $\mathrm{KB}$ is a signaling pathway common to IL-1R/TLRs, including IL-33 and ST2 (12), and NF-KB plays a role in cardiac hypertrophy (16), we explored the role of NF-KB activation in the antihypertrophic effect of IL-33. Quantitative analyses from 5 separate experiments in cardiomyocytes (Figure 3A) and cardiac fibroblasts (Figure $3 \mathrm{~B}$ ) with or without angiotensin II $(1 \mu \mathrm{M})$, phenylephrine $(50 \mu \mathrm{M})$, or IL-33 $(10 \mathrm{ng} / \mathrm{ml})$ confirmed that IL-33, angiotensin II, and phenylephrine independently activated NF-KB in both cell types. However, the activation of NF-kB by angiotensin II and phenylephrine in cardiomyocytes was attenuated by IL-33; this effect was only transiently observed in cardiac fibroblasts. As shown by quantitative analyses from 4 separate experiments in cardiomyocytes (Figure 3C) and cardiac fibroblasts (Figure 3D), angiotensin II and IL-33 induced inhibitor of NF- $\mathrm{KB} \alpha$ (IKB $\alpha$ ) phosphorylation in both cell types, but IL-33 reduced IкB $\alpha$ phosphorylation in cardiomyocytes, consistent with the EMSA results. Transient transfection reporter assays $(n=3$ mice per group) also demonstrated that angiotensin II increased NF- $\mathrm{KB}$ promoter activity $(66 \% \pm 26 \% ; P<0.05$; data not shown) and that cotreatment with IL-33 abolished the induction of NF-KB promoter activity $(8 \% \pm 12 \%$; $P=\mathrm{NS}$; data not shown). Furthermore, cotreatment with $\mathrm{SST} 2$ released the repression of NF- $\kappa \mathrm{B}$ promoter activity by IL-33 ( $42 \% \pm 5 \%$; $P<0.05$; data not shown). Interestingly, the reduction in both NF-KB nuclear binding activity and I $\mathrm{B} \beta \alpha$ phosphorylation by IL-33 was not observed under stimulation with PDGF-BB $(10 \mathrm{ng} / \mathrm{ml})$ or TNF- $\alpha$ (10 ng/ml), which also activate NF-KB (Figure 3E). These data demonstrate a dual nature of IL-33 signaling in cardiomyocytes, with mild activation of NF-KB but attenuation of NF-KB by other hypertrophic stimuli.

IL-33 can also activate MAPK pathways (14). In cardiomyocytes, IL-33 increased phosphorylation of ERK, p38, and JNK MAPKs (Figure 3F). IL-33-induced activation of MAPK pathways was generally modest compared with IL-1 $\beta$ in cardiomyocytes. Interestingly, cotreatment with IL-33 did not affect ERK phosphorylation, but decreased p38 MAPK and JNK phosphorylation induced by angiotensin II. IL-33 minimally activated Akt and did not impair angiotensin IIinduced Akt activation (Figure 3F). These data suggest that IL-33 may have antihypertrophic properties through suppression of agonist-induced MAPK pathways, but the effects on MAPK sig- 
A
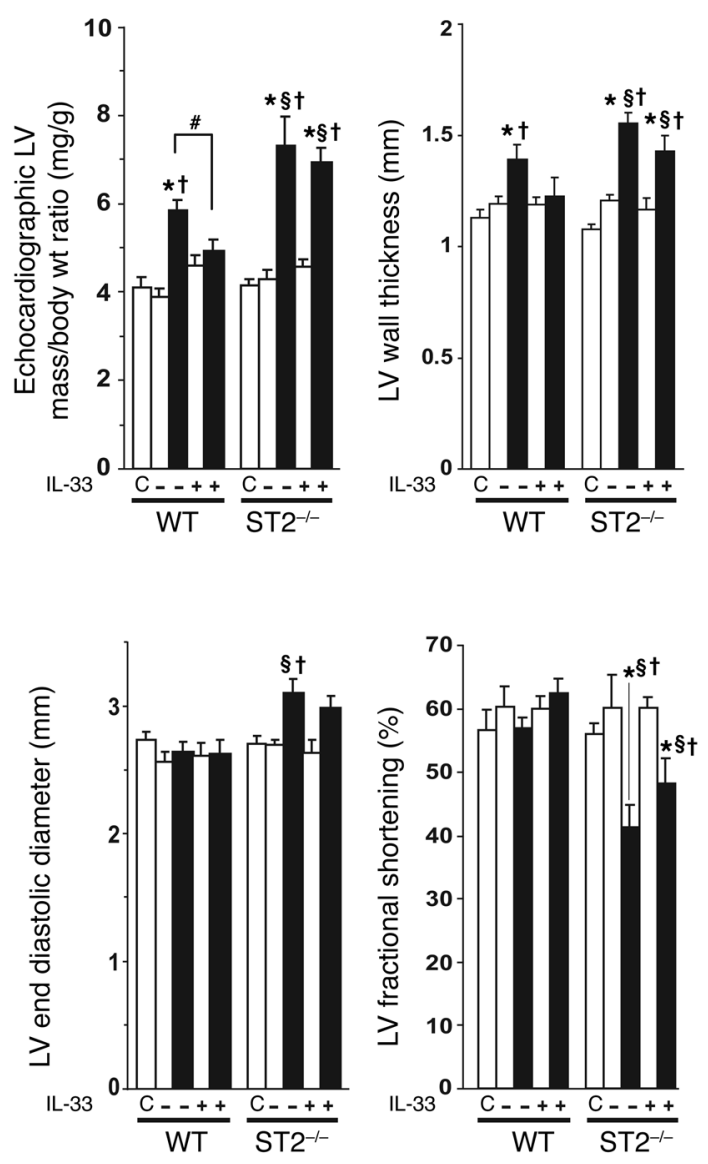

B
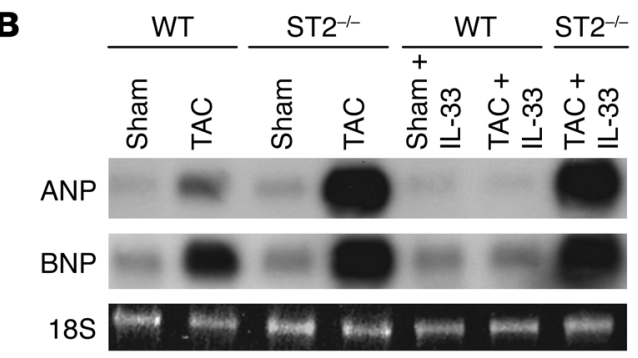

C

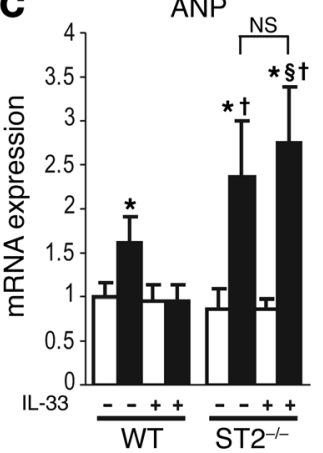

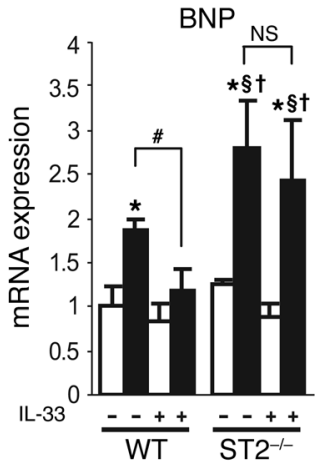

D

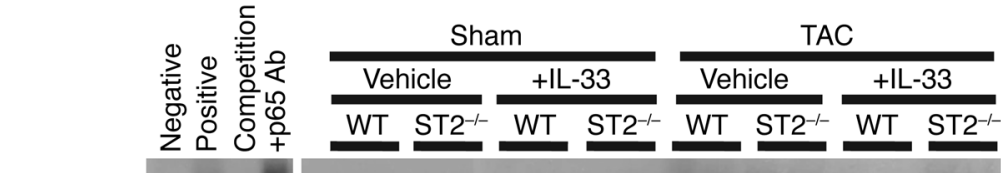

Supershift

$\mathrm{NF}-\kappa \mathrm{B}$

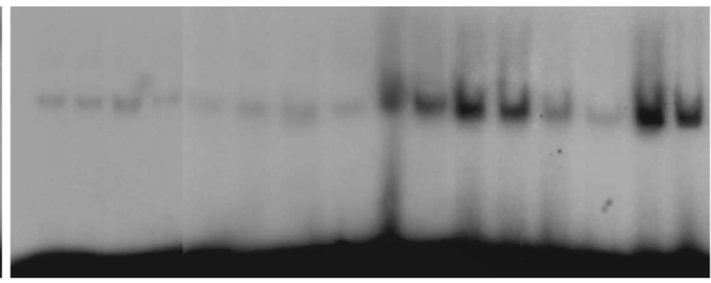

\section{Figure 5}

IL-33/ST2 signaling is cardioprotective in vivo. (A) Echocardiographic analysis at 4 weeks after operation demonstrated increased left ventricular mass, left ventricular wall thickness, and reduced fractional shortening in ST2 $2^{-/}$mice. Treatment with IL-33 reduced hypertrophy only in WT mice. IL-33 caused no significant change under non-stress conditions in vivo. $n=10$ (nonoperated control); 8 (WT sham); 10 (WT TAC); 8 (WT sham + IL-33); 10 (WT TAC + IL-33); 8 (ST2 ${ }^{-1-}$ sham); 12 (ST2 ${ }^{-1-T A C) ; ~} 8$ (ST2-/- sham + IL-33); and 10 (ST2 ${ }^{-1-}$ TAC + IL-33). (B) Representative images and (C) quantitative analysis of mRNA expression of ANP and BNP relative to internal control (18S) in the left ventricle at 1 week after operation, as assessed by Northern analysis. White and black bars indicate sham-operated and TAC, respectively. (D) NF-kB activation from EMSA in vivo 1 week after operation. ANP and BNP expression and NF-kB activity increased in ST2 $2^{-/-}$mice compared with WT mice; IL-33 reversed these changes only in WT mice. Positive and negative control mixtures as well as specific competition mixtures and supershift induce by 065 antibody are also shown. ${ }^{*} P<0.05$ versus nonoperated control $(\mathbf{A})$ or sham-operated WT $(\mathbf{C})$; $\$ P<0.05$ versus the same treatment in WT; ${ }^{\dagger} P<0.05$ versus sham in the same group; ${ }^{P} P<0.05$.

naling was less dramatic compared with the effects of IL-33 on $\mathrm{NF}-\kappa \mathrm{B}$ activation.

Because hypertrophic stimuli such as angiotensin II can signal through the generation of ROS (17), we also performed experiments to determine whether IL-33 regulates angiotensin II-mediated ROS generation. As shown in Figure 3, G and H, we observed that IL-33 significantly decreased the generation of ROS in the presence of angiotensin II or phenylephrine.

ST2 signaling is cardioprotective in vivo. The above experiments demonstrated that IL-33 was antihypertrophic in vitro and that sST2 inhibited IL-33 signaling through ST2L. Prior human studies have demonstrated that increased SST2 is an adverse prognostic sign in cardiovascular disease $(8,9)$. This raises the hypothesis that IL-33 signaling through ST2L is cardioprotective in vivo. To explore this hypothesis, we performed genotype-blinded TAC in ST2 $2^{-/-}$mice versus WT littermate controls. The ST2-/- mouse has deletion of most of exons 4 and 5 of the ST2 gene and thus lacks both ST2L and sST2; the mouse develops normally, although it is deficient in T helper cell type 2 cytokine responses (18).

Histological studies (Figure 4A) demonstrated increased cardiac fibrosis and increased cardiomyocyte cross-sectional area after TAC in ST2 ${ }^{-/-}$mice $(n=12)$ compared with WT littermates $(n=10$; Figure 4B). Furthermore, administration of purified IL-33 $(2 \mu \mathrm{g} / \mathrm{d}$ i.p. for 4 weeks) reduced cardiac fibrosis and cardiomyocyte hypertrophy in WT mice, but not in ST2-/- littermates (as expected, since the IL-33 ligand should not affect mice without the ST2L receptor unless there is another, as-yet unknown IL-33 receptor). Gross heart/body weight measurements were consis- 
A
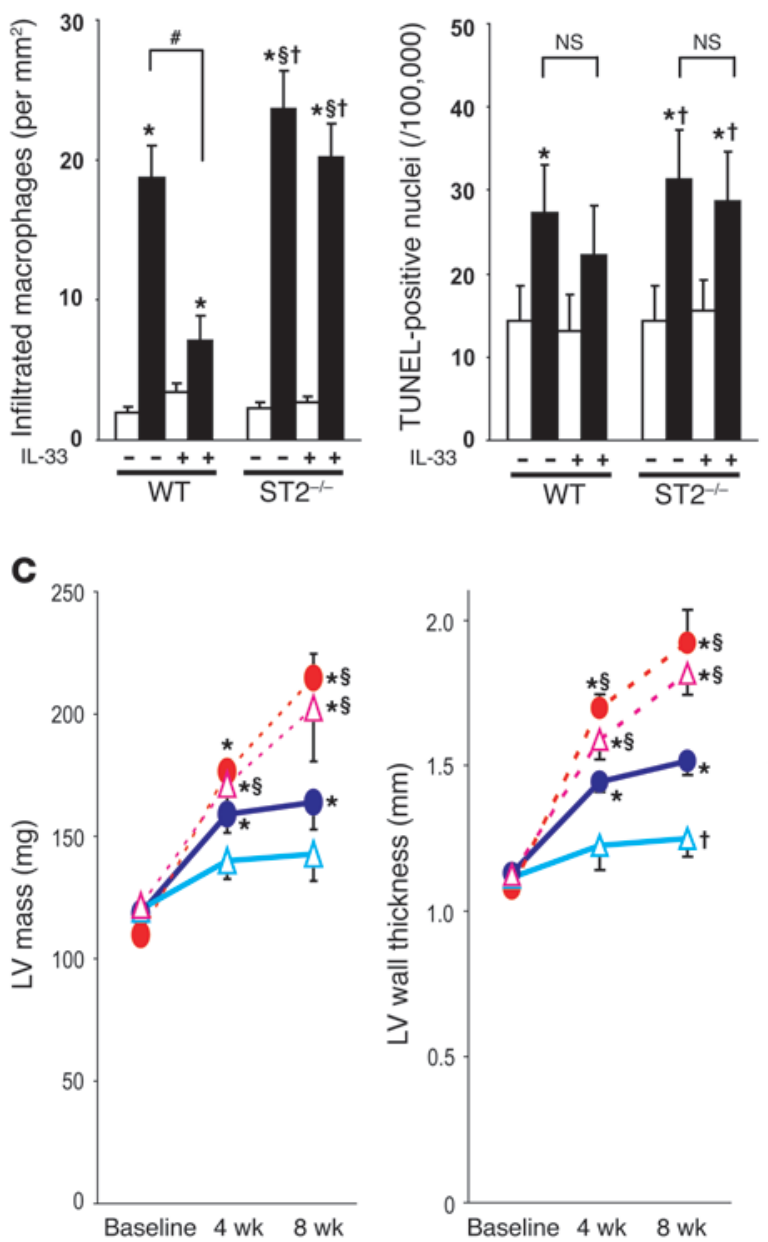

B
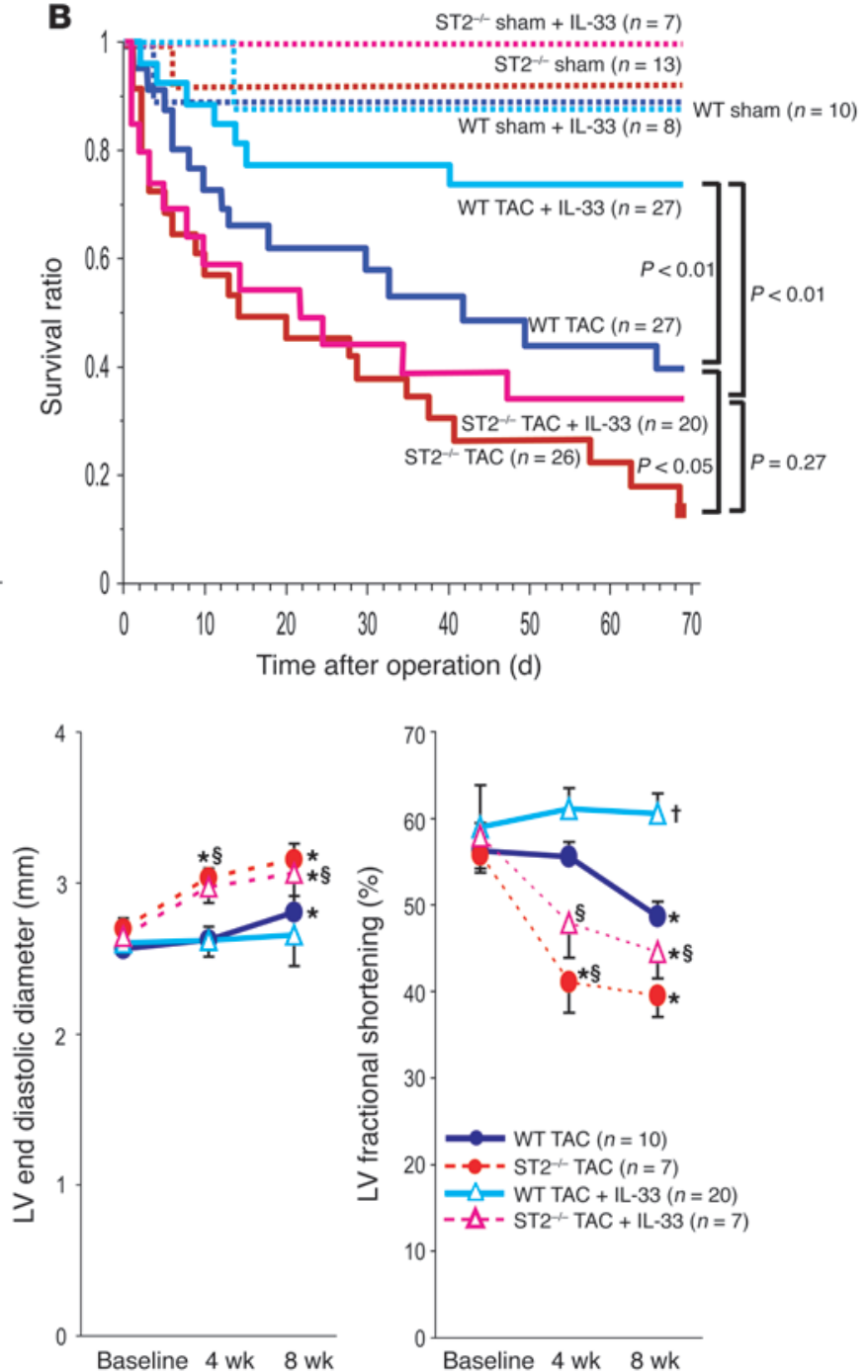

Figure 6

IL-33 improves survival after TAC and reduces TAC-induced macrophage infiltration, but does not inhibit apoptosis in vivo. TAC was performed on WT and ST2 $2^{--}$littermates. (A) Quantitative analysis of macrophages and TUNEL stain-positive nuclei. Computer-based image analysis was used. TAC increased macrophage infiltration after 1 week of operation. IL-33 alone did not induce macrophage infiltration in either WT or ST2-/- mice but co-treatment reduced macrophage infiltration after TAC only in WT mice. TAC approximately doubled the number of TUNEL-positive nuclei after 4 weeks in both WT and ST2 ${ }^{-1-}$ mice. IL-33 treatment did not affect TUNEL positivity. (B) Kaplan-Meier survival curve analysis revealed that the survival of ST2 $2^{--}$mice under TAC was significantly reduced compared with that of WT mice. This experiment was blinded so that all procedures were performed without knowledge of mouse genotype. (C) Serial echocardiographic analysis of surviving mice revealed that ST2 ${ }^{-1-}$ mice had increased left ventricular mass and left ventricular wall thickness and reduced contractile function compared with WT mice. ${ }^{*} P<0.05$ versus shamoperated WT $(\mathbf{A})$ or baseline $(\mathbf{C}) ;{ }^{\circledR} P<0.05$ versus the same treatment in WT; ${ }^{\dagger} P<0.05$ versus Sham in the same group; ${ }^{\#} P<0.05$.

tent with histomorphometric data, revealing an antihypertrophic effect of IL-33 in WT mice but not in ST2-/- mice (Figure 4C). In contrast, no change in any parameter was observed among nonoperated control $(n=7)$, sham-operated WT $(n=8)$, and shamoperated ST2 $2^{--}(n=8)$ groups.

A separate echocardiographic analysis (also blinded to genotype) was performed in these animals. These data demonstrated that deletion of ST2 caused both increased hypertrophy and impaired systolic function following TAC (Figure 5A). In addition, treatment with IL-33 reduced hypertrophy in WT mice, but not in ST2 $/-$ littermates. Increased left ventricular dilation and reduced fractional shortening were observed only in ST2-/- mice after TAC, and these changes were not reversed by treatment with IL-33 in ST2-/- mice (Figure 5A).

Following TAC, ST2 ${ }^{-/}$mice had increased gene expression of atrial and B-type natriuretic peptides (ANP and BNP; Figure 5, B and C), genes commonly induced by hypertrophic stimuli. Treatment with IL-33 markedly attenuated induction of both genes in WT but not in ST2-/- mice. Quantitative gene expression analysis confirmed that deletion of ST2 signaling enhanced the induction of BNP by TAC and that treatment with IL-33 effectively reversed the induction of these hypertrophic genes in WT mice, but not ST2 $2^{-/-}$mice, with TAC (Figure 5C). Consistent with the in vitro experiments described above, TAC-enhanced NF- $\kappa \mathrm{B}$ nuclear binding activity 
A

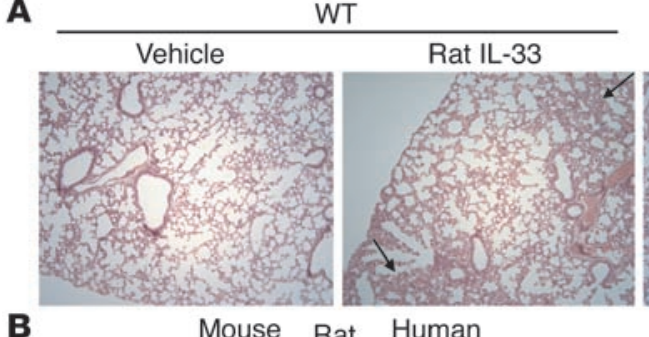

WT

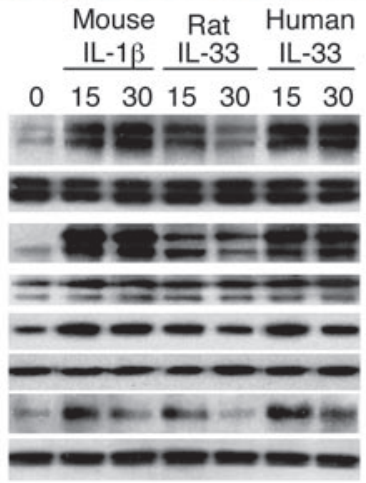

\section{Time Dose} (min) $\quad(\mathrm{ng} / \mathrm{ml})$

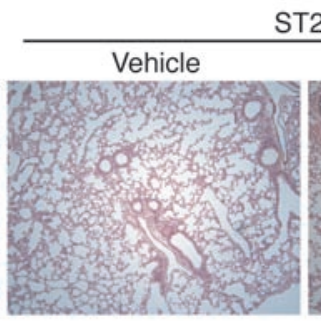
$\mathrm{ST}^{-1-}$

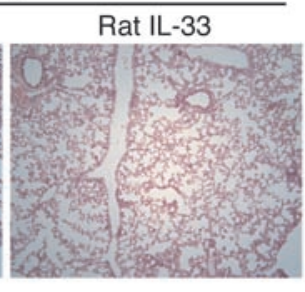

Mouse IL-1 $\beta$ Rat IL-33 Human IL-33 $0 \longdiv { 1 1 0 1 0 0 } 1 1 0 1 0 0 1 0 0 0 1 1 0 1 0 0 1 0 0 0$

\begin{tabular}{|c|}
\hline $\mathrm{p}$-ERK \\
\hline ERK \\
\hline p-JNK \\
\hline JNK \\
\hline p-p38 \\
\hline p38 \\
\hline$-p 65-N F-\kappa B$ \\
\hline p65-NF-кB \\
\hline
\end{tabular}

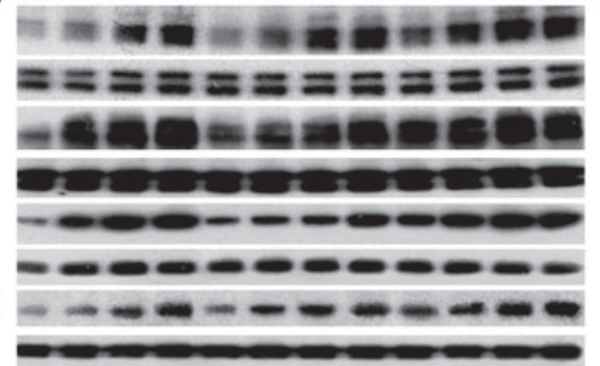

\section{Figure 7}

Rat IL-33 has weaker potency than human IL-33 and causes focal pulmonary inflammation in mice. (A) Representative specimens of lung (H\&E stain; original magnification, $\times 100)$ from mice 1 week after sham operation with or without 7 days' treatment with rat IL-33 $(2 \mu \mathrm{g} / \mathrm{d})$. In the pulmonary parenchyma of WT mice, IL-33 treatment led to mild focal infiltrations of inflammatory cells (arrows) within and adjacent to vessels. This was not seen in vehicle-treated mice. These changes were not observed in ST2 $2^{-/-}$mice with or without IL-33 treatment. (B) Time- and dose-dependent activation of ERK, JNK, p38, and p65-NF-KB by mouse IL-1 $\beta$ and recombinant mature rat or human IL-33 are shown. Mature rat IL-33 showed relatively weaker activation of MAPKs and NF-KB than the other proteins.

was prominently observed in left ventricular myocardia of both WT and ST2 $-/$ - mice. IL-33 inhibited NF- $\mathrm{KB}$ induction only in WT mice and not in $\mathrm{ST}^{-/-}$mice (Figure 5D). Because some previous reports showed regional myocardial infiltration of inflammatory cells in experimental studies with mice under pressure overload $(19,20)$, we evaluated macrophage infiltration within myocardium after 1 week of treatment in both phenotypes. As shown in Figure 6A, IL-33 did not significantly increase macrophage infiltration in $\mathrm{WT}$ or ST2 $2^{-/-}$mice, including perivascular regions. However, mice with TAC showed significant increases in macrophage infiltration, which was decreased by cotreatment with IL-33 in WT mice. Furthermore, to investigate the mechanism of cardiac histological and functional changes, we also evaluated the presence of apoptosis within myocardium 4 weeks after operation with or without IL-33 treatment. There was an increase in the percentage of TUNEL-positive stained nuclei in mice that underwent TAC (Figure 6A), as previously reported $(21,22)$. The percentage of TUNEL-positive cells was not changed by treatment with IL-33 or by genetic deletion of ST2 (Figure 6A). These data suggest that changes in apoptosis do not directly cause the changes in cardiac fibrosis.

In a separate blinded and randomized experiment, the role of IL-33/ST2 signaling in mortality after TAC was studied. Mortality was increased after TAC in ST2 ${ }^{-/}$mice $(n=26)$ compared with WT littermates ( $n=27$; Figure 6B). Furthermore, IL-33 significantly improved survival after TAC in WT mice $(n=27)$, but not in ST2 $/$ - littermates $(n=20)$. Echocardiographic studies were also performed on each mouse, and these data on mice that survived for 8 weeks after TAC indicated that ST2 $/-$ mice $(n=10)$ had a greater increase in left venways remained unchanged. None of these changes were observed in ST2-/- mice with or without IL-33 treatment (Figure 7A), suggesting that the mild pulmonary inflammation is due to IL-33/ST2 signaling. To further explore the difference in the inflammatory response in contrast with the prior report (12), we compared activation of intracellular signaling pathways by mouse IL- $1 \beta$ (positive control) with that by different amounts of rat and human IL-33 at different time points (Figure 7B) on mouse NIH3T3 cells. Both rat and human IL-33 activated ERK, JNK, p38, and NF-кB. Surprisingly, there was a trend toward more intense activation by human IL-33.

\section{Discussion}

Although ST2 is a potentially useful biomarker in heart diseases such as heart failure (8) and myocardial infarction (9), the pathophysiological role of ST2 in the myocardium has been unclear, largely due to the lack of a known ligand for ST2. Here we showed that the recently described ligand IL-33 was synthesized by cardiac fibroblasts and abrogated angiotensin II- and phenylephrineinduced hypertrophy in cardiomyocytes in vitro. Furthermore, targeted deletion of ST2 in mice enhanced cardiac hypertrophy and fibrosis following mechanical overload and impaired contractility and survival, while administration of purified recombinant IL-33 improved pathological changes and survival in WT mice, but not in ST2 ${ }^{-/}$mice. Therefore, these data demonstrate that IL-33/ST2 signaling protects the myocardium under mechanical overload.

IL-1R/TLR superfamily receptors share similar downstream signaling pathways (23), including binding with adaptor proteins such as myeloid differentiation primary-response pro- 
tein-88 (MyD88) and its variant, Mal; recruiting TNF receptor-associated factor-6 (TRAF6); and activating apoptosis signal-regulated kinase-1 (MEKKK, to activate p38MAPK; ref. 24) and NF-кB-inducing kinase-1 (NIK1, to activate IкB kinases; ref. 25 ) as well as TGF- $\beta$-activated kinase-1 (TAK1), which leads to activation of p38 MAPK (26), JNK (25) or NF-кB $(25,26)$. Because NF- $\mathrm{B}$ plays a pivotal role in cardiomyocyte hypertrophy in vitro $(27,28)$ and in vivo $(28,29)$, we studied the activation of NF- $\mathrm{KB}$ under both basal and stimulated conditions. IL-33 mildly activated NF- $\kappa \mathrm{B}$ in basal conditions, but IL-33 markedly attenuated angiotensin II- and phenylephrineinduced NF- $\kappa \mathrm{B}$ activation in cardiomyocytes.

Regulation of NF- $\mathrm{KB}$ activation may explain the antihypertrophic effect of IL-33. The mechanisms by which IL-33 may dampen NF- $\kappa \mathrm{B}$ activation are unclear. Our experiments suggest that IL-33 can suppress ROS generation by angiotensin II or phenylephrine, and ROS generation has been implicated in NF- $\mathrm{KB}$ activation (17). Alternatively, feedback inhibition (30) and oscillation (31) are commonly found with NF- $\mathrm{KB}$ activation, so that other mechanisms for the antihypertrophic effect of IL-33 are also plausible. Angiotensin II $(28,32)$ as well as other $G$ protein-coupled receptor (GPCR) ligands (33) promote cardiac hypertrophy induced by mechanical pressure overload $(26,28,29,32,34)$. Interestingly, accumulating evidence suggests a relationship between signals induced by GPCR (especially angiotensin II) and those induced by the IL-1R/TLR superfamily in activating NF-кB and MAPKs in cardiomyocytes. For example, the small GTPase Rac1 $(35,36)$, which is translocated to the cytoplasmic membrane and activated through angiotensin II receptors (35), subsequently activates NADPH oxidases that produce ROS (36), which regulate further downstream kinases. Li et al. (37) reported that Rac1 facilitates the recruitment of NADPH oxidases into active endosomal MyD88/ IL-1R1 complexes and subsequent redox-dependent recruitment of TRAF6 to the MyD88/IL-1R1 complex. Furthermore, depletion of TLR4 (29) or inactivation of MyD88 (34) decreases cardiac hypertrophy induced by TAC.

Using a higher dose and a longer treatment period, we observed similar, but reduced, pulmonary inflammation compared with the initial description of IL-33 by Schmitz et al. (12). While IL-33 has proinflammatory effects in the lung, we did not observe increased macrophage or eosinophil infiltration in myocardium after IL-33 treatment. However, IL-33 reduced macrophage infiltration after TAC in WT mice, raising the hypothesis that IL-33 may be antiinflammatory and antifibrotic in myocardium through a primary effect on cardiomyocytes or other cardiac cells. In our study, we used purified mature rat IL-33 protein in mice, while the prior study used the recombinant human protein in mice. We generated mature rat and human IL-33 proteins by the same methods, and the direct comparison of potency between our recombinant rat and human IL-33 on mouse cells indicated weaker activation of MAPKs or NF- $\mathrm{KB}$ by mature rat IL-33 compared with mature human IL-33. This may explain why we observed less pulmonary inflammation than did Schmitz et al., who used human IL-33 in mice (12). The mature mouse IL-33 amino acid sequence (GenBank accession no. AY905582) is 90\% identical to that of rat IL-33 (GenBank accession no. BC081993), but only $57 \%$ identical to that of human IL-33 (GenBank accession no. AY905581), so the differences in inflammation may be due to differences between the human and rat IL-33 proteins or differences in the preparation or the genetic background of mice.
Fibrosis is a hallmark of long-standing myocardial overload, and the relationship between cardiac fibrosis and cardiomyocyte pathophysiology remains incompletely understood. Recent reports suggest that cardiac fibrosis might be regulated by signaling upstream of NF- $\mathrm{KB}$ (such as TAK1) in cardiomyocytes $(26,34)$ or by cross-talk with cardiomyocytes through cytokines (38). We speculate that patients with increased sST2 may be inhibiting endogenous IL-33 and ST2 cardioprotection through the binding of myocardial IL-33 to sST2, thereby preventing signaling through ST2L. There was a trend toward increased amino acid uptake due to sST2 in cardiomyocytes also treated with angiotensin II or phenylephrine. This could be due to a direct effect of sST2, as has been previously suggested (39). Alternatively, in these experiments, sST2 could have reduced endogenous IL-33 signaling. Although cardiomyocytes appear to synthesize less IL-33 than do cardiac fibroblasts, it is possible that endogenous IL-33 suppresses angiotensin II- or phenylephrine-induced amino acid uptake. This concept is supported by the trend toward increasing leucine uptake by the anti-ST2 antibody in the presence of angiotensin II. In addition, we observed that PMA caused a dose-dependent robust induction in both sST2 and IL-33 protein expression, with a dose-dependent decrease in free IL-33. This further supports the concept of sST2-bound sequestration of IL-33 that could decrease physiological activity of IL-33 on cells through a decoy action of sST2. Although our data are consistent with cardioprotective IL-33 signaling and inhibition of cardioprotection by sST2 as a decoy receptor, it is also possible that $\mathrm{SST} 2$ may serve as a reservoir for IL-33. This scenario has been described for soluble IL-6 receptor (40). Thus, sST2 may function as more than an inhibitor of ST2L signaling. Detailed characterization of the relative binding affinities of native sST2 and ST2L for IL-33 has not yet been performed. This characterization will be important for future understanding of the role of specific levels of sST2 as a decoy receptor. Soluble IL receptors can have substantially lower affinity to the same ligand compared with membrane-bound isoforms (41), and thus substantial excess of sST2 may be necessary to inhibit IL-33 signaling through ST2L. Although these data demonstrate that myocardial cells express IL-33 and that IL-33 signaling can be cardioprotective, these experiments do not exclude a role for IL-33 or sST2 expression by noncardiac cells, including leukocytes.

There was a modest increase in NF- $\mathrm{KB}$ binding in the ventricles of ST2 $2^{--}$mice treated with IL-33. Although our data generally suggest no effect of IL-33 in the absence of ST2, we cannot conclusively exclude the possibility of another function of IL-33, other than binding to ST2. Finally, it is possible that sST2 is not only a biomarker for poor outcome, but also a true pathophysiological mediator of disease progression. This hypothesis will require further exploration of the quantitative relationships between serum sST2 levels and myocardial IL-33 paracrine signaling.

\section{Methods}

All reagents are from Sigma-Aldrich, otherwise noted. We expressed and purified IL-33 proteins for all of the experiments described using affinity chromatography as described in Production of recombinant rat and human IL-33 protein and polyclonal anti-rat IL-33 antibody.

ST2 $-/$ mice. Targeted ST2-disrupted mice were maintained on a $129 \times$ C57BL/ 6 background as described previously (18). All animal procedures were conducted in accordance with guidelines published in the NIH Guide for the Care and Use of Laboratory Animals and were approved by the Harvard Medical School Standing Committee on Animals. 
Cell culture and cyclic mechanical strain. Cell culture of rat neonatal cardiomyocytes and cardiac fibroblasts was performed as previously described $(42,43)$. Briefly, hearts from 1- to 3-day-old Sprague-Dawley rat neonates (Charles River Laboratories) were digested with trypsin $(1 \mathrm{mg} / \mathrm{ml}$; Invitrogen $)$ and collagenase II $(0.8 \mathrm{mg} / \mathrm{ml}$; Worthington Biochemical Corp.) and plated on noncoated culture dishes for 1.5 hours. Attached cells were further cultured with Dulbecco's modified essential medium (Invitrogen) containing 10\% fetal bovine serum (Invitrogen) and used as cardiac fibroblasts after 2 passages. Nonattached cells were replated on $0.1 \%$ gelatin-precoated dishes and further cultured with $7 \%$ fetal bovine serum as cardiomyocytes. Cells were serum-starved for 24 hours prior to experiments. Biomechanical strain of cells was performed as previously described $(6,44)$. For in vitro cell size analysis, cardiomyocytes were plated at $30 \%-40 \%$ confluence to allow definition of cell borders. Cell culture of mouse NIH3T3 cells was performed using Dulbecco's modified essential medium (Invitrogen) containing 10\% fetal calf serum (Invitrogen).

Quantitative PCR. mRNA expression was analyzed by quantitative PCR using the QuantiTect SYBR Green reverse transcription-PCR kit (QIAGEN) and the Light Cycler System (Roche Applied Science) as previously described (45). Total RNA was extracted from cells with TRI reagent, and $0.2 \mu \mathrm{g}$ of RNA was analyzed. Sequences of PCR primers were as follows: rat IL-33, 5'-TCGCACCTGTGACTGAAATC-3' and 5'-ACACAGCATGCCACAAACAT-3'; rat ANP, 5'-ATACAGTGCGGTGTCCAACA-3' and $5^{\prime}$-CGAGAGCACCTCCATCTCTC-3'; rat BNP, 5'-GGAAATGGCTCAGAGACAGC-3' and 5'-CGATCCGGTCTATCTTCTGC-3'.

Northern analysis. Purified RNA from adult rat brains was used for the synthesis of cDNA with reverse transcriptase-PCR. The primer set for the synthesis of the 471-base pair IL-33 cDNA probe, the 245-base pair sST2 cDNA probe, and the 242-base pair mouse BNP cDNA probe were as follows: IL-33, 5' -AGTATCCAAGGAACTTCACTGCTA-3' and 5' -TTACATCTTAGAGAGCTTAAACATGAT-3'; sST2, 5' -TTACCCAGCCAGGATGTTTC-3' and 5'-CTAGGGGCTTGGCTTCTCTT-3'; rat ANP, 5'-ATACAGTGCGGTGTCCAACA-3' and 5'-CGAGAGCACCTCCATCTCTC-3'; mouse BNP, 5' -CAGCTCTTGAAGGACCAAGG- $3^{\prime}$ and $5^{\prime}$-AGACCCAGGCAGAGTCAGAA-3'. Northern analysis was performed as previously described (44). Autoradiograms were quantified by densitometry (Scion Image 4.0), and mRNA levels were normalized to densitometry of $18 \mathrm{~S}$ or $28 \mathrm{~S}$ ribosomal RNA ethidium bromide staining.

Production of recombinant rat and human IL-33 protein and polyclonal antirat $I L-33$ antibody. The GenBank accession numbers for rat and human IL-33 are BC081993 and AY905581, respectively. Rat or human IL-33 cDNA was subcloned into the expression vector pTrcHis-TOPO (Invitrogen), starting with amino acid 109 or 112 of the full-length rat or human protein, respectively, with an $\mathrm{N}$-terminal His tag. After sequencing, One-shot TOP10 bacteria (Invitrogen) were transformed, and expression was induced with Isopropyl $\beta$-D-1-thiogalactopyranoside. Four hours after induction, bacteria were harvested and the pellet was resuspended in B-PER Bacterial Protein Extraction Reagent (Pierce Biotechnology) with EDTA-free protease inhibitor cocktail. The lysate was purified with Ni-chelating affinity chromatography using a Ni-NTA Purification system (Invitrogen). Briefly, Ni-NTA beads were washed twice after batch binding with $40 \mathrm{ml}$ of $20 \mathrm{mM}$ imidazole, $300 \mathrm{mM} \mathrm{NaCl}$, and $50 \mathrm{mM}$ potassium phosphate, $\mathrm{pH}$ 8.0. IL-33 was eluted with $10 \mathrm{ml}$ of $250 \mathrm{mM}$ imidazole, $300 \mathrm{mM}$ $\mathrm{NaCl}$, and $50 \mathrm{mM}$ potassium phosphate, $\mathrm{pH}$ 8.0. IL-33 protein was further concentrated, dialyzed, and purified by high-performance liquid chromatography (BioLogic DuoFlow System; Bio-Rad) using a HiPrep sephacryl size exclusion column (GE HealthCare) with buffer containing $150 \mathrm{mM} \mathrm{NaCl}$ and $50 \mathrm{mM}$ potassium phosphate, $\mathrm{pH}$ 7.2. Endotoxin level $(<0.03 \mathrm{EU} / \mu \mathrm{g}$ protein) was determined by Limulus Amebocyte Lysate PYROGENT Ultra (Cambrex) as previously described (12). Using this protein, we raised a polyclonal anti-rat IL-33 antibody in rabbits (Maine Biotechnology) and purified the antibody with protein-A affinity chromatography. Pulldown assay between recombinant IL-33 with His tag and mouse ST2L-Fc chimera (R\&D Systems) was performed as described previously (12) with some modification, i.e., using Ni-NTA beads (Invitrogen) and anti-HisG antibody (Invitrogen) instead of agarose-bound avidin D and streptavidin-HRP conjugate, respectively, followed by appropriate secondary HRP-conjugated antibody (Bio-Rad) reaction for 1 hour at room temperature and enhanced chemiluminescence.

$\left[{ }^{3} \mathrm{H}\right]$-Leucine incorporation assay. Leucine incorporation in rat neonatal cardiomyocytes was detected as previously described (46). Cells were treated with $1.0 \mu \mathrm{Ci} / \mathrm{ml}\left[{ }^{3} \mathrm{H}\right]$-leucine (PerkinElmer) for 24 hours, washed, and fixed with $10 \%$ trichloroacetate for 45 minutes at $4{ }^{\circ} \mathrm{C}$, after which radioactivity was determined by liquid scintillation counting (Beckman Coulter). We used rat anti-mouse ST2L monoclonal antibody (R\&D Systems) as a functional neutralizing antibody, as reported previously (12). We also used normal rat IgG2a and human IL-1R-related protein 2-Fc chimera (R\&D Systems) as controls for rat anti-mouse ST2L monoclonal antibody and human SST2-Fc chimera (R\&D Systems), respectively.

Fluorescence microscopy. Immunofluorescence staining on paraffinembedded mouse hearts or rat neonatal cardiomyocyte cell culture was performed as described previously $(42,45)$. In addition to rabbit polyclonal anti-IL-33 antibody (diluted 1:100; Maine Biotechnology), mouse monoclonal (anti-vimentin, diluted 1:100; Abcam; ref. 42) and goat polyclonal (anti-discoidin domain receptor-2, diluted 1:50; Santa Cruz Biotechnology Inc.; refs. 4,15 ) antibodies were used to detect fibroblasts in separate specimens. Samples were visualized using an inverted fluorescent microscope (IX-70; Olympus). For measuring cardiomyocyte size, the surface area of the cells was calculated with ImagePro Express software. Cells from randomly selected fields were analyzed.

Immunohistochemistry and TUNEL analysis. Formalin-fixed, paraffin-embedded sections were prepared as previously described (42). For immunohistochemistry, the first antibodies used were anti-cleaved caspase-3 (Cell Signaling Technology) and anti-mac3 (BD Biosciences). For TUNEL analysis, the In Situ apoptosis detection kit (Chemicon) was used according to the manufacturer's protocol with some optimal modifications on the treatment periods to reduce reaction time with $D A B$ within a minute.

EMSA. EMSAs were performed as described previously (47). Cells were lysed for 10 minutes on ice in $10 \mathrm{mM}$ HEPES ( $\mathrm{pH} 7.6$ ), $15 \mathrm{mM} \mathrm{KCl}, 2 \mathrm{mM}$ $\mathrm{MgCl} 2,0.1 \mathrm{mM}$ EDTA, $1 \mathrm{mM}$ DTT, $0.5 \mathrm{mM}$ PMSF, $10 \mu \mathrm{g} / \mathrm{ml}$ leupeptin, and $0.2 \%$ Nonident P-40. Dishes were gently scraped, and nuclei were collected by centrifugation at $800 \mathrm{~g}$ for 30 seconds and then suspended in a solution of $50 \mathrm{mM}$ HEPES, $400 \mathrm{mM} \mathrm{KCl}, 0.1 \mathrm{mM}$ EDTA, $10 \%$ glycerol, $1 \mathrm{mM}$ DTT, $0.5 \mathrm{mM}$ PMSF, and $10 \mu \mathrm{g} / \mathrm{ml}$ leupeptin. The mixture was incubated on ice for 30 minutes, and the supernatant was collected after centrifugation for 15 minutes at $400 \mathrm{~g}$. Binding reactions were performed with 5 or $10 \mu \mathrm{g}$ of nuclear protein in $10 \mathrm{mM}$ Tris, $1 \mathrm{mM}$ DTT, $1 \mathrm{mM}$ EDTA, $5 \%$ glycerol, $0.1 \%$ Triton X-100, $1 \mathrm{mg}$ poly(dIdC), $5 \mu \mathrm{g}$ BSA, and $10,000 \mathrm{cpm}$ of $\left[{ }^{32} \mathrm{P}\right]$-labeled oligonucleotide. The specific NF- $\mathrm{KB}$ consensus oligonucleotides were 5'-AGTTGAGGGGACTTTCCCAGGC-3' and 5'-GCCTGGGAAAGTCCCCTCAACT-3' (Promega). Positive and negative control mixtures as well as specific competition mixtures were made as recommended by the manufacturer, using binding buffer with positive

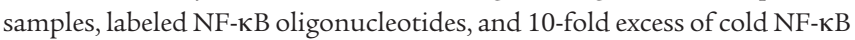
consensus oligonucleotides for specific competition. DNA complexes were separated on a $6 \%$ nondenaturing polyacrylamide gel in Tris- $\mathrm{HCl}(6.7 \mathrm{mM})$, $\operatorname{EDTA}(1 \mathrm{mM})$, and ammonium acetate $(3.3 \mathrm{mM})$.

$N F-\kappa B$ luciferase assay. Neonatal rat cardiomyocytes were transfected using Lipofectamine Plus (Invitrogen) with a luciferase reporter plasmid for $\mathrm{NF}-\kappa \mathrm{B}$ and with $\beta$-galactosidase as described previously (47). After 48 
hours of transfection, cells were then stimulated for an additional 24 hours, lysed, treated with Dual-Light assay system (Applied Biosystems), and analyzed using a luminometer (PerkinElmer).

Oxidative stress assay. We performed an assay to detect oxidative stress as reported previously (48). Cardiomyocytes were incubated with 2,7-dichlorodihydrofluorecein diacetate (DCFDA; Molecular Dynamics Inc.) for 45 minutes and washed in phosphate-buffered saline, and fluorescence intensity was measured using a fluorometer (PerkinElmer) at $595 \mathrm{~nm}$.

Immunoprecipitation. Immunoprecipitation was performed as described previously (45) with some modifications. Briefly, after washing with PBS, protein $\mathrm{G}$-agarose beads were presaturated with a far-excessive amount of human sST2-Fc protein (R\&D Systems) at $4^{\circ} \mathrm{C}$ overnight and then washed 3 times with PBS. Conditioned media $(1 \mathrm{ml})$ with or without $20 \mu \mathrm{g} \mathrm{sST2}-\mathrm{Fc}$ protein preincubation was then incubated with presaturated beads for 2 hours at room temperature.

Western analysis. Western analyses were performed as described previously (45). Membranes (PVDF; PerkinElmer) were incubated overnight with primary antibodies anti-p44/42 MAPK, diluted 1:500; phosphorylated p44/42 MAPK (Thr202/Tyr204), diluted 1:1,000; phosphorylated JNK (Thr183/Tyr185), diluted 1:500; p38 MAPK, diluted 1:500; phosphorylated p38 MAPK (Thr180/Tyr182), diluted 1:500; phosphorylated Akt (Ser473),

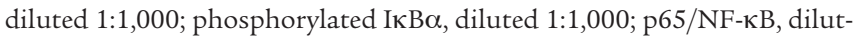
ed 1:1,000; and phosphorylated p65/NF-кB, diluted 1:1,000 (all from Cell Signaling Technology); anti-mouse ST2L, diluted 1:1,000 (R\&D Systems); and anti-IL-33, diluted 1:500 (Maine Biotechnology) at $4{ }^{\circ} \mathrm{C}$, detected with HRP-conjugated antibodies (Bio-Rad) and enhanced chemiluminescence.

TAC and treatment with IL-33 protein. Targeted ST2 ${ }^{-/}$mice and their WT littermates were used for the experiments. TAC was produced as described previously $(49,50)$ on 8 - to 10 -week-old mice. All operative procedures were performed by a single operator with over 20 years' rodent cardiac surgery experience who was blinded to genotype and the randomized treatment assignment. Following the procedure, each mouse received daily i.p. injection of recombinant rat IL-33 $(2 \mu \mathrm{g})$ or vehicle from the day after TAC induction until the day of harvest.

Echocardiography. Echocardiographic acquisition and analysis were performed by an echocardiographer blinded to treatment group. Light anesthesia with spontaneous respiration was achieved with intraperitoneal pentobarbital $(10 \mu \mathrm{g} / \mathrm{g})$. All images were taken at a heart rate greater than 400 beats per minute to minimize effects of anesthesia, using a Sonos-4500 echocardiography system (Philips) and a 15-MHz transducer (Philips). The average of 3 consecutive cardiac cycles was used. Left ventricular mass was calculated by the M-mode method (51). Left ventricular fractional shortening was calculated as a percentage as follows: $(\mathrm{EDD}-\mathrm{ESD}) / \mathrm{EDD} \times 100$, where EDD is end-diastolic dimension and ESD is end-systolic dimension. The calculated and actual weights (taken at autopsy) had excellent correlation $(r=0.88 ; P<0.0001 ; n=50$ mice from 1 and 4 weeks after TAC).

Histological analysis. A planimetry image analysis program developed in our laboratory was used by an investigator blinded to treatment group to quantitate the 30 high-power field regions randomly selected in each slice with H\&E stain (for cross-sectional area) or Sirius red stain (for fibrotic area). The cross-sectional area of cardiomyocytes was measured as described previously (52). Percent fibrotic area was calculated as (red area/total area) $\times 100$. The scores from all slices were averaged to calculate their respective values.

Statistics. Data are expressed as mean \pm SEM. Statistical significance was determined using 2-tailed Student's $t$ test or ANOVA with Fisher's post-hoc test as appropriate. A $P$ value less than 0.05 was considered statistically significant.

\section{Acknowledgments}

We acknowledge Joseph Gannon and Catherine MacGillivray for expert technical support. Shoji Sanada was supported by a Japan Society for the Promotion of Science research fellowship. This study was supported by a grant from the National Heart, Lung, and Blood Institute, NIH (R01HL081404 to R.T. Lee).

Received for publication October 12, 2006, and accepted in revised form February 27, 2007.

Address correspondence to: Richard T. Lee, Partners Research Facility, 65 Landsdowne Street, Cambridge, Massachusetts 02139, USA. Phone: (617) 768-8272; Fax: (617) 768-8270; E-mail: rlee@partners.org.

Shoji Sanada and Daihiko Hakuno contributed equally to this work.
1. Sadoshima, J., and Izumo, S. 1997. The cellular and molecular response of cardiac myocytes to mechanical stress. Annu. Rev. Physiol. 59:551-571.

2. McKinsey, T.A., and Olson, E.N. 2005. Toward transcriptional therapies for the failing heart: chemical screens to modulate genes. J. Clin. Invest. 115:538-546. doi:10.1172/JCI200524144.

3. Diez, J., Gonzalez, A., Lopez, B., and Querejeta, R. 2005. Mechanisms of disease: pathologic structural remodeling is more than adaptive hypertrophy in hypertensive heart disease. Nat. Clin. Pract. Cardiovasc. Med. 2:209-216.

4. Baudino, T., Carver, W., Giles, W.R., and Borg, T.K. 2006. Cardiac fibroblasts: friend or foe? Am.J. Physiol. Heart Circ. Physiol. 291:H1015-H1026.

5. Grossman, W., Jones, D., and McLaurin, L.P. 1975. Wall stress and patterns of hypertrophy in the human left ventricle. J. Clin. Invest. 56:56-64.

6. Weinberg, E.O., et al. 2002. Expression and regulation of ST2, an interleukin-1 receptor family member, in cardiomyocytes and myocardial infarction. Circulation. 106:2961-2966.

7. Iwahana, H., et al. 1999. Different promoter usage and multiple transcription initiation sites of the interleukin-1 receptor-related human ST2 gene in UT-7 and TM12 cells. Eur. J. Biochem. 264:397-406

8. Weinberg, E.O., et al. 2003. Identification of serum soluble ST2 receptor as a novel heart failure biomarker. Circulation. 107:721-726.

9. Shimpo, M., et al. 2004. Serum levels of the interleukin- 1 receptor family member ST2 predict mortality and clinical outcome in acute myocardial infarction. Circulation. 109:2186-2190.

10. Kumar, S., Minnich, M.D., and Young, P.R. 1995 ST2/T1 protein functionally binds to two secreted proteins from Balb/c 3T3 and human umbilical vein endothelial cells but does not bind interleukin 1. J. Biol. Chem. 270:27905-27913.

11. Gayle, M.A., et al. 1996. Cloning of a putative ligand for the T1/ST2 receptor. J. Biol. Chem. 271:5784-5789.

12. Schmitz, J., et al. 2005. IL-33, an interleukin-1-like cytokine that signals via the IL-1 receptor-related protein ST2 and induces T helper type 2-associated cytokines. Immunity. 23:479-490.

13. Manabe, I., Shindo, T., and Nagai, R. 2002. Gene expression in fibroblasts and fibrosis: involvement in cardiac hypertrophy. Circ. Res. 91:1103-1113.

14. Marian, A.J. 2000. Pathogenesis of diverse clinical and pathological phenotypes in hypertrophic cardiomyopathy. Lancet. 355:58-60.

15. Goldsmith, E.C., et al. 2004. Organization of fibroblasts in the heart. Dev. Dyn. 230:787-794.

16. Kawano, S., et al. 2005. Blockade of NF-kappaB ameliorates myocardial hypertrophy in response to chronic infusion of angiotensin II. Cardiovasc. Res. 67:689-698.

17. Hirotani, S., et al. 2002. Involvement of nuclear factor-kappaB and apoptosis signal-regulating kinase 1 in G-protein-coupled receptor agonistinduced cardiomyocyte hypertrophy. Circulation. 105:509-515.

18. Townsend, M.J., Fallon, P.G., Matthews, D.J., Jolin, H.E., and McKenzie, A.N. 2000. T1/ST2-deficient mice demonstrate the importance of T1/ST2 in developing primary $\mathrm{T}$ helper cell type 2 responses. J. Exp. Med. 191:1069-1076.

19. Kuster, G.M., et al. 2005. Mineralocorticoid receptor inhibition ameliorates the transition to myocardial failure and decreases oxidative stress and inflammation in mice with chronic pressure overload. Circulation. 111:420-427.

20. Shioi, T., et al. 1997. Increased expression of interleukin- 1 beta and monocyte chemotactic and activating factor/monocyte chemoattractant protein-1 in the hypertrophied and failing heart with pressure overload. Circ. Res. 81:664-671.

21. Okada, K., et al. 2004. Prolonged endoplasmic reticulum stress in hypertrophic and failing heart after aortic constriction: possible contribution of endoplasmic reticulum stress to cardiac myocyte 
apoptosis. Circulation. 110:705-712.

22. Yang, G., et al. 2001. Cyclosporine reduces left ventricular mass with chronic aortic banding in mice, which could be due to apoptosis and fibrosis. J. Mol. Cell. Cardiol. 33:1505-1514.

23. Dunne, A., and O'Neill, L.A. 2003. The interleukin-1 receptor/Toll-like receptor superfamily: signal transduction during inflammation and host defense. Sci. STKE. 2003:re3.

24. Matsuzawa, A., et al. 2005. ROS-dependent activation of the TRAF6-ASK1-p38 pathway is selectively required for TLR4-mediated innate immunity. Nat. Immunol. 6:587-592.

25. Ninomiya-Tsuji, J., et al. 1999. The kinase TAK1 can activate the NIK-I kappaB as well as the MAP kinase cascade in the IL-1 signalling pathway. Nature. 398:252-256.

26. Zhang, D., et al. 2000. TAK1 is activated in the myocardium after pressure overload and is sufficient to provoke heart failure in transgenic mice. Nat. Med. 6:556-563.

27. Purcell, N.H., et al. 2001. Activation of NF-kappa $\mathrm{B}$ is required for hypertrophic growth of primary rat neonatal ventricular cardiomyocytes. Proc. Natl. Acad. Sci. U. S. A. 98:6668-6673.

28. Freund, C., et al. 2005. Requirement of nuclear factor-kappaB in angiotensin II- and isoproterenolinduced cardiac hypertrophy in vivo. Circulation. 111:2319-2325.

29. Ha, T., et al. 2005. Reduced cardiac hypertrophy in toll-like receptor 4-deficient mice following pressure overload. Cardiovasc. Res. 68:224-234.

30. Hoffmann, A., and Baltimore, D. 2006. Circuitry of nuclear factor kappaB signaling. Immunol. Rev. 210:171-186.

31. Nelson, D.E., et al. 2004. Oscillations in NF-kappaB signaling control the dynamics of gene expression. Science. 306:704-708.

32. Rockman, H.A., Wachhorst, S.P., Mao, L., and Ross, J.J. 1994. ANG II receptor blockade prevents ventricular hypertrophy and ANF gene expression with pressure overload in mice. Am. J. Physiol. 266:H2468-H2475.
33. Rapacciuolo, A., et al. 2001. Important role of endogenous norepinephrine and epinephrine in the development of in vivo pressure-overload cardiac hypertrophy. J. Am. Coll. Cardiol. 38:876-882.

34. Ha, T., et al. 2006. Blockade of MyD88 attenuates cardiac hypertrophy and decreases cardiac myocyte apoptosis in pressure overload-induced cardiac hypertrophy in vivo. Am. J. Physiol. Heart Circ. Physiol. 290:H985-H994.

35. Hingtgen, S.D., et al. 2006. Nox2-containing NADPH oxidase and Akt activation play a key role in angiotensin II-induced cardiomyocyte hypertrophy. Physiol. Genomics. 26:180-191.

36. Nishida, M., et al. 2005. G alpha 12/13- and reactive oxygen species-dependent activation of c-Jun $\mathrm{NH} 2$-terminal kinase and p38 mitogen-activated protein kinase by angiotensin receptor stimulation in rat neonatal cardiomyocytes. J. Biol. Chem. 280:18434-18441.

37. Li, Q., et al. 2006. Nox2 and Rac1 regulate $\mathrm{H} 2 \mathrm{O} 2$ dependent recruitment of TRAF6 to endosomal interleukin-1 receptor complexes. Mol. Cell. Biol. 26:140-154.

38. Sarkar, S., Vellaichamy, E., Young, D., and Sen, S. 2004. Influence of cytokines and growth factors in ANG II-mediated collagen upregulation by fibroblasts in rats: role of myocytes. Am. J. Physiol. Heart Circ. Physiol. 287:H107-H117.

39. Saccani, S., Polentarutti, N., Penton-Rol, G., Sims, J.E., and Mantovani, A. 1998. Divergent effects of LPS on expression of IL-1 receptor family members in mononuclear phagocytes in vitro and in vivo. Cytokine. 10:773-780.

40. May, L.T., Ndubuisi, M.I., Patel, K., and Garcia, D. 1995. Interleukin-6 chaperones in blood. Ann. N. Y. Acad. Sci. 762:120-128.

41. Economides, A.N., et al. 2003. Cytokine traps: multi-component, high-affinity blockers of cytokine action. Nat. Med. 9:47-52.

42. Hsieh, P.C., Davis, M.E., Gannon, J., MacGillivray, C., and Lee, R.T. 2006. Controlled delivery of PDGF-BB for myocardial protection using injectable self-assembling peptide nanofibers. J. Clin.
Invest. 116:237-248. doi:10.1172/JCI25878.

43. Yokoyama, T., et al. 1999. Angiotensin II and mechanical stretch induce production of tumor necrosis factor in cardiac fibroblasts. Am. J. Physiol. 276:H1968-H1976.

44. Yamamoto, K., et al. 1999. Induction of tenascin$\mathrm{C}$ in cardiac myocytes by mechanical deformation. Role of reactive oxygen species. J. Biol. Chem. 274:21840-21846.

45. Hakuno, D., Takahashi, T., Lammerding, J., and Lee, R.T. 2005. Focal adhesion kinase signaling regulates cardiogenesis of embryonic stem cells. J. Biol. Chem. 280:39534-39544.

46. Yamamoto, K., Ohki, R., Lee, R.T., Ikeda, U., and Shimada, K. 2001. Peroxisome proliferator-activated receptor gamma activators inhibit cardiac hypertrophy in cardiac myocytes. Circulation. 104:1670-1675.

47. Frantz, S., et al. 1999. Toll4 (TLR4) expression in cardiac myocytes in normal and failing myocardium. J. Clin. Invest. 104:271-280.

48. Schulze, P.C., et al. 2004. Hyperglycemia promotes oxidative stress through inhibition of thioredoxin function by thioredoxin-interacting protein. J. Biol. Chem. 279:30369-30374.

49. Rockman, H.A., et al. 1991. Segregation of atrialspecific and inducible expression of an atrial natriuretic factor transgene in an in vivo murine model of cardiac hypertrophy. Proc. Natl. Acad. Sci. U. S. A. 88:8277-8281.

50. Tanaka, N., et al. 1996. Transthoracic echocardiography in models of cardiac disease in the mouse. Circulation. 94:1109-1117.

51. Collins, K.A., et al. 2001. Accuracy of echocardiographic estimates of left ventricular mass in mice. Am. J. Physiol. Heart Circ. Physiol. 280:H1954-H1962.

52. Takemoto, M., et al. 1997. Important role of tissue angiotensin-converting enzyme activity in the pathogenesis of coronary vascular and myocardial structural changes induced by long-term blockade of nitric oxide synthesis in rats. J. Clin. Invest. 99:278-287. 